\title{
NASAL COLONIZATION OF METHICILLIN-RESISTANT STAPHYLOCOCCUS AUREUS AMONG CLINICAL POSTGRADUATES IN A COASTAL KARNATAKA MEDICAL COLLEGE - PREVALENCE AND ANTIBIOGRAM PATTERN
}

\author{
ISHA JUNEJA, ASHWINI HEGDE, POOJA RAO, RADHAKRISHNA M* \\ Department of Microbiology, Kasturba Medical College, Manipal Academy of Higher Education, Mangalore, Karnataka, India. \\ Email: manipuraradhakrishna@yahoo.com
}

Received: 05 June 2018, Revised and Accepted: 28 June 2018

\section{ABSTRACT}

Objective: The incidence of community-acquired and hospital-acquired Staphylococcus aureus infections has been rising with increasing emergence of drug-resistant strains called methicillin-resistant $S$. aureus (MRSA). The prime objective of the study was to ascertain the prevalence of carriage rate of MRSA among the clinical postgraduate students of a medical college of Coastal Karnataka, India.

Methods: A total of 133 clinical postgraduates have participated in the present study. Swabs from both anterior nares were collected and processed for $S$. aureus from the consenting participants after giving a set of questionnaires. The isolated strains of $S$. aureus were screened for methicillin resistance by the modified Kirby-Bauer method using cefoxitin ( $30 \mu \mathrm{g})$ disc. Antibiotic susceptibility testing for all isolates of $S$. aureus was also done against other antibiotics. E test was used to know vancomycin resistance for MRSA.

Results: The numbers of S. aureus isolated of 133 participants were 22 (16.5\%). Of 22 isolates of S. aureus, 12 were MRSA (54.5\%). The MRSA carriage rate of clinical postgraduates of a medical college from Coastal Karnataka, India, was 9\%. All the MRSA isolates were susceptible to vancomycin and teicoplanin. Contact with chronic patients and consumption of antibiotics in past 6 months were found to be statistically significant as the risk factor associated with the acquisition of $S$. aureus/MRSA carriage status among the participants. No vancomycin-resistant $S$. aureus was reported from the present study.

Conclusion: The S. aureus and MRSA nasal carriage rates of clinical postgraduates recorded in this study were significantly high when compared with the general population. This could be due to their continuous exposure to patients in clinical settings. It is desirable that these students must receive sufficient knowledge regarding control measures to avoid the spread of MRSA infection in hospitals.

Keywords: Staphylococcus aureus, Methicillin-resistant Staphylococcus aureus, Nasal carriage, Clinical postgraduates.

(c) 2018 The Authors. Published by Innovare Academic Sciences Pvt Ltd. This is an open access article under the CC BY license (http://creativecommons. org/licenses/by/4. 0/) DOI: http://dx.doi.org/10.22159/ajpcr.2018.v11i10.27713

\section{INTRODUCTION}

Staphylococcus aureus, in particular, methicillin-resistant S. aureus (MRSA) has gained prominence all over the world. There is hospitalacquired MRSA (HA-MRSA) as well as community-acquired MRSA (CA-MRSA) strains and infections. MRSA nosocomial infection (NI) outbreaks and prevalence among various populations have been well reported in literature particularly for developed countries. MRSA strains are resistant to a cluster of antibiotics, and because of the same reason, the treatment of infections due to this organism is always a challenge, more so when the critically ill patients are infected. Hospital infection due to MRSA is a known cause of increased hospital stay, cost, morbidity, and mortality especially among the critically ill. Asymptomatically colonized patients, and health-care providers are the primary sources of MRSA in the hospital environment, with the latter being more commonly notified as links in the transmission of MRSA between patients.

S. aureus is one of the most famous human bugs, capable of causing a wide range of infections. Even though primary infections are not familiar, a great deal of the virulence occurs through cross-infection in hospitals and other institutional settings. Whereas, individuals with good health have a small risk of contracting an invasive infection caused by $S$. aureus, whereas they can be carriers of the organism [1]. Because its primary habitat is moist squamous epithelium of the anterior nares, most invasive $S$. aureus infections are believed to arise from the nasal carriage [2]. The overall incidence of CA and HA $S$. aureus infections has been rising with increasing emergence of drug-resistant strains called as MRSA [3]. Earlier limited to hospitals, MRSA infections have been increasingly reported in the community nowadays [4]. One of the recent meta-analysis of 27 studies of the prevalence of CA-MRSA among hospital patients that used clinical specimens, conducted at the time of admission, yielded a prevalence of MRSA of $30.2 \%$ [5]. A diverse of studies have examined community prevalence of nasal carriage of $S$. aureus in various subpopulations, such as adult outpatients, health care workers, college students, and injection drug users [6]. The overall prevalence of $S$. aureus ranges from $20 \%$ to $45 \%$, with an estimate of MRSA colonization from 10 community surveillance studies was $1.3 \%$ [5]. These data are quite useful to clinicians when trying to decide the likelihood that a given patient has a Staphylococcal infection and, if so, whether antibiotic coverage should be provided for resistant strains. Exposure to microbes is an inherent risk of working in patient care settings. Given its increasing incidence in the general population, acquisition of MRSA is a particular concern for healthcare workers $[7,8]$.

The medical course pattern varies from country to country. In a study of university students of Brazil, the nasal carriage of $S$. aureus was $40.8 \%(102 / 250)$. Of the isolates, six isolates $(5.8 \%)$ were methicillin-resistant and carried the mecA gene suggestive of CAMRSA in Brazil [9]. The study including medical students of Taiwanese University revealed overall S. aureus and MRSA carriage rate of $19.3 \%$ and $2.2 \%$, respectively, without any significant difference between the pre-clinical and clinical students regarding nasal carriage of $S$. aureus and MRSA [10]. Another cross-sectional study enrolling the whole 
medical student population $(\mathrm{n}=387)$ from Colombia, showed the carriage rates MSSA and MRSA of $25 \%$ and $1.6 \%$, respectively [11]. Another cross-sectional study from the medical students of Texas University including 203 participants concluded that 60 (29.6\%) were $S$. aureus carriers and 15 (7.4\%) were MRSA, respectively. Hospitalization in the past 12 months and recent skin infection were significantly associated with the risk of being an MRSA carrier [12]. In one of the studies at University students of Malaysia comprised 209 medical students both pre and clinical showed 21(10\%) S. aureus without any MRSA as nasal carriage but not associated with any risk factor significantly [13]. A cross-sectional study involving 450 medical students of Winston-Salem, North Carolina revealed 29\% of $S$. aureus carriage rate and $2 \%$ of MRSA. Older male volunteers suffering from persistent sinusitis and not taking antibiotics were at higher risk for carrying $S$. aureus [6]. Literature survey also revealed a US-based study where 182 students of $1^{\text {st }}, 2^{\text {nd }}$, and $3^{\text {rd }}$ years of medical course with $34 \%$ and $2.7 \%$ of $S$. aureus and MRSA carriage, respectively, without an occupational hazard of patient care exposure in $3^{\text {rd }}$-year medical students [14].

Postgraduate students with the clinical specialization in medical institutions comprise a unique population at risk for MRSA acquisition as they are always in touch with patients in the hospital and clinics. This study has been designed to see the nasal carriage status among the Clinical Postgraduates of our institution. At the same time, this study might help us to highlight any significant relation, if present, between the specific clinical departments such as surgery, medicine and obstetrics and the carriage of the organism among the Clinical Postgraduates of our institution.

\section{METHODS}

The proposed study was conducted in the microbiology diagnostic laboratory of a medical college of Coastal Karnataka, India.

\section{Study design}

The said work was a type of cross-sectional study. A sample size of 168 was calculated considering the power of $80 \%$, the confidence level of $95 \%$, and a relative precision $6 \%$, and prevalence of nasal carriage of MRSA to be $21 \%$, and a non-response rate of $10 \%$ [6].

Table 1: Gender-wise distribution of participants, carriage status of $S$. aureus and MRSA

\begin{tabular}{llll}
\hline Gender & $\begin{array}{l}\text { Number of } \\
\text { participants(\%) }\end{array}$ & $\begin{array}{l}\text { S. aureus } \\
\text { carriers(\%) }\end{array}$ & $\begin{array}{l}\text { MRSA } \\
\text { carriers (\%) }\end{array}$ \\
\hline Male & $80(60.15)$ & $16(72.73)$ & $6(50)$ \\
Female & $53(39.85)$ & $6(27.27)$ & $6(50)$ \\
Total & 133 & 22 & 12 \\
\hline
\end{tabular}

S. aureus: Staphylococcus aureus, MRSA: Methicillin-resistant

Staphylococcus aureus
The formula used was

$\mathrm{N}=4 \mathrm{pq} / \mathrm{d}^{2}$

Where, $p$ is prevalence, $q$ is $1-p$, then $d$ is relative precision

All consenting clinical postgraduates were randomly recruited into the study. The study proposal was submitted to the Ethical Review Committee of the Institution for approval. The age, sex, lifestyle, current skin infection/other illness, particulars of clinical specialty and additional relevant information about the participants were obtained in a pro forma designed for this purpose. The proposed study was carried out between June 1, and August 31, 2015. Of 241 clinical postgraduates, 133 did participate in the study. Swabs from both anterior nares of consenting persons were taken with a sterile swab stick moistened with sterile physiological saline. Processing of the samples was done immediately within $2 \mathrm{~h}$ after collection. In case of delay, more than $2 \mathrm{~h}$ the swabs were stored at $4^{\circ} \mathrm{C}$ for maximum $24 \mathrm{~h}$. The swabs were inoculated onto Mannitol Salt Agar (MSA), and the inoculated MSA was incubated at $37^{\circ} \mathrm{C}$ for $18-24 \mathrm{~h}$. The growth of the organism was identified as $S$. aureus using standard tests, such as colony morphology, Gram stain, catalase test, and coagulase test [15]. The isolated strains of $S$. aureus were screened for methicillin resistance by the modified Kirby-Bauer method using cefoxitin (30 $\mu \mathrm{g})$ disc on Mueller-Hinton agar (MHA) [16]. The MHA on which cefoxitin disc was placed was incubated aerobically at $37^{\circ} \mathrm{C}$ for $18 \mathrm{~h}$. Isolates with inhibition zone diameter $\leq 21 \mathrm{~mm}$ around cefoxitin disc were considered as MRSA strains [17]. Screening for vancomycin susceptibility was also done by the modified KirbyBauer method using vancomycin disc $(30 \mu \mathrm{g})$ on MHA incubated at $37^{\circ} \mathrm{C}$ for $24 \mathrm{~h}$. Vancomycin-resistant was determined MIC determination by E test. Antibiotic sensitivity to all $S$. aureus isolates against other antibiotics such as penicillin, amoxicillin/clavulanic acid, erythromycin, clindamycin, chloramphenicol, cotrimoxazole, ceftriaxone, gentamycin, linezolid, teicoplanin, and ciprofloxacin were determined by the modified Kirby-Bauer method. All inoculum on MHA was 4-6 h growth of pure isolates in Mueller-Hinton Broth, with the density equivalent to a 0.5 McFarland turbidity standard. Controls strains such as S. aureus ATCC 25923, MRSA ATCC 29213, and MSSA ATCC 33591 were used. Antibiotic discs were procured from HiMedia Laboratories Pvt., Ltd., India. Antibiotic sensitivity testing and interpretation of the result were done according to CLSI guidelines [18].

\section{Statistical analysis}

Results were compelled, tabulated and all data were subjected to the statistical package Statistical Package for the Social Sciences version 17.0. The outcomes were presented in the form of tables and graphs. Associations of different factors were done using Chi-square test. $p$ - value $<0.005$ was considered as significant.

Table 2: Participants' parameters: Department and PG year wise distributions

\begin{tabular}{lllll}
\hline Department & $\mathbf{1}^{\text {st }}$ year & $\mathbf{2}^{\text {nd }}$ year & $\mathbf{3}^{\text {rd }}$ year & Total numbers (\%) \\
\hline Orthopedics & 2 & 4 & 3 & $9(6.8)$ \\
E.N.T. & 5 & 2 & 4 & $11(8.3)$ \\
Dermatology & 1 & 0 & 2 & $3(2.3)$ \\
Medicine & 12 & 12 & 10 & $34(25.56)$ \\
Surgery & 4 & 3 & 3 & $10(7.5)$ \\
Ophthalmology & 4 & 4 & 5 & $13(9.8)$ \\
Pediatrics & 6 & 9 & 6 & $4(15.78)$ \\
O.B.G. & 2 & 1 & 1 & $9(6.8)$ \\
Audiology & 9 & 0 & 0 & $1(0.8)$ \\
Psychiatry & 0 & 0 & 3 & $8(6)$ \\
Anesthesia & 2 & 3 & 0 & $7(5.3)$ \\
Physiotherapy & 3 & 4 & 1 & $3(2.3)$ \\
Radiology & 1 & 1 & $39(29.32)$ & 133 \\
Total n=133 & $51(38.35)$ & $43(32.33)$ & &
\end{tabular}




\section{RESULTS}

Of 241, a total of 133 clinical postgraduates participated in the said study. Among the 133 participants, those were screened for nasal carriage of S. aureus. 80 (60.15\%) were males, and 53 (39.85\%) were females (Table 1). The department wise and year wise categorization of the participants were shown in Table 2. The types of organisms isolated from the anterior nares of the participants were shown in Table 3. Coagulase-negative Staphylococcus (CoNS) was found to be predominant 84 (63.16\%) followed by S. aureus 22 (16.54\%). Of 133, 22 participants were positive for nasal carriage of $S$. aureus giving a carriage rate of 16.54\% (Fig. 1). 12 of 22 isolates of S. aureus turned out to be MRSA with the pace of $54.54 \%$ (Fig. 2). Overall, MRSA nasal carriage rate was $9 \%$ in this study (Fig. 3). The antibiotic susceptibility pattern of S. aureus was shown in Fig. 4. Usage of cefoxitin disc was found to be convenient over oxacillin disc in the determination of MRSA. Of the 22 isolates of S. aureus, $95.5 \%$ were resistant to penicillin, and $81.8 \%$ were resistant to ciprofloxacin whereas resistance to erythromycin was $54.55 \%$. Eventually, all S. aureus strains were $100 \%$ susceptible to vancomycin, and teicoplanin and $95 \%$ sensitive to linezolid (Fig. 4). The MRSA isolate was $100 \%$ responsive to vancomycin, teicoplanin and $91.67 \%$ sensitive to linezolid (Fig. 5). Maximum numbers of MRSA were found in the postgraduates of pediatrics (4) followed by surgery (3) department (Table 4). Year wise distribution of MRSA carriers was shown in Table 5. It was observed that of 12 , eight strains of MRSA were isolated from $1^{\text {st }}$. year students. The association between S. aureus/MRSA carriage status and the various factors was illustrated in Figs. 6 and 7. Contact with the

Table 3: Nature of organisms isolated from anterior nares

\begin{tabular}{ll}
\hline Organism isolated & Number (\%) \\
\hline No growth & $18(13.53)$ \\
CoNS & $84(63.16)$ \\
S. aureus & $22(16.54)$ \\
Diphtheroids & $4(3.007)$ \\
Candida & $5(3.76)$ \\
Total (n=133) & $133(100)$ \\
\hline
\end{tabular}

CoNS: Coagulase-negative Staphylococcus, S. aureus: Staphylococcus aureus

Table 4: Carriage status of $S$. aureus and MRSA among the postgraduates of various clinical departments

\begin{tabular}{lllll}
\hline Department & S. aureus (\%) & MRSA (\%) & Others & Total \\
\hline Orthopedics & $4(18.2)$ & $1(8.3)$ & $5(4.5)$ & 9 \\
E.N.T. & $0(0)$ & $0(0)$ & $11(9.9)$ & 11 \\
Dermatology & $1(4.5)$ & $0(0)$ & $2(1.8)$ & 3 \\
Medicine & $4(18.2)$ & $2(16.7)$ & $30(27)$ & 34 \\
Surgery & $3(13.6)$ & $3(25)$ & $7(6.3)$ & 10 \\
Ophthalmology & $0(0)$ & $0(0)$ & $13(11.7)$ & 13 \\
Pediatrics & $6(27.3)$ & $4(33.3)$ & $15(13.5)$ & 21 \\
O.B.G. & $1(4.5)$ & $1(8.3)$ & $3(2.7)$ & 4 \\
Audiology & $1(4.5)$ & $1(8.3)$ & $8(7.2)$ & 9 \\
Psychiatry & $0(0)$ & $0(0)$ & $1(0.9)$ & 1 \\
Anesthesia & $1(4.5)$ & $0(0)$ & $7(6.3)$ & 8 \\
Physiotherapy & $0(0)$ & $0(0)$ & $7(6.3)$ & 7 \\
Radiology & $1(4.5)$ & $0(0)$ & $2(1.8)$ & 3 \\
Total & 22 & 12 & 111 & 133 \\
\hline
\end{tabular}

S. aureus: Staphylococcus aureus, MRSA: Methicillin-resistant

Staphylococcus aureus

Table 5: Carriage status of $S$. aureus and MRSA among the postgraduates in various years

\begin{tabular}{lllll}
\hline P.G. & S. aureus (\%) & MRSA (\%) & Others (\%) & Total \\
\hline $1^{\text {st }}$ & $12(54.5)$ & $8(66.7)$ & $39(35.1)$ & 51 \\
$2^{\text {nd }}$ & $6(27.3)$ & $3(25)$ & $37(33.3)$ & 43 \\
$3^{\text {rd }}$ & $4(18.2)$ & $1(8.3)$ & $35(31.5)$ & 39 \\
\hline
\end{tabular}

S. aureus: Staphylococcus aureus, MRSA: Methicillin-resistant

Staphylococcus aureus patient with chronic infection and consumption of antibiotic in past 6 months were found to be associated with MRSA carriage status.

\section{DISCUSSION}

The anterior nares of humans found to be the primary ecological niches of the $S$. aureus [19]. The carriage patterns are of three types. Approximately $20 \%$ of the individuals almost always carry one kind

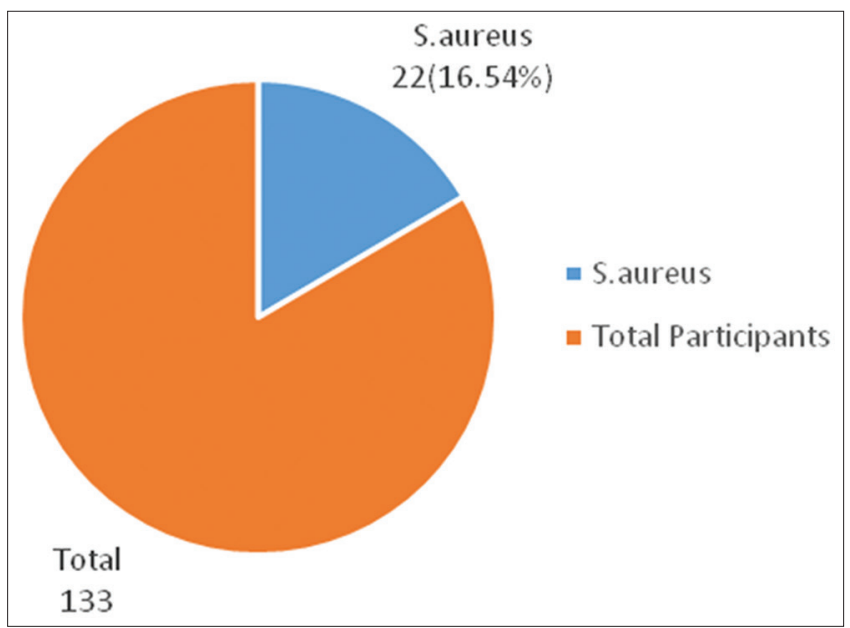

Fig. 1: Percentage of Staphylococcus aureus carriers among the total participants

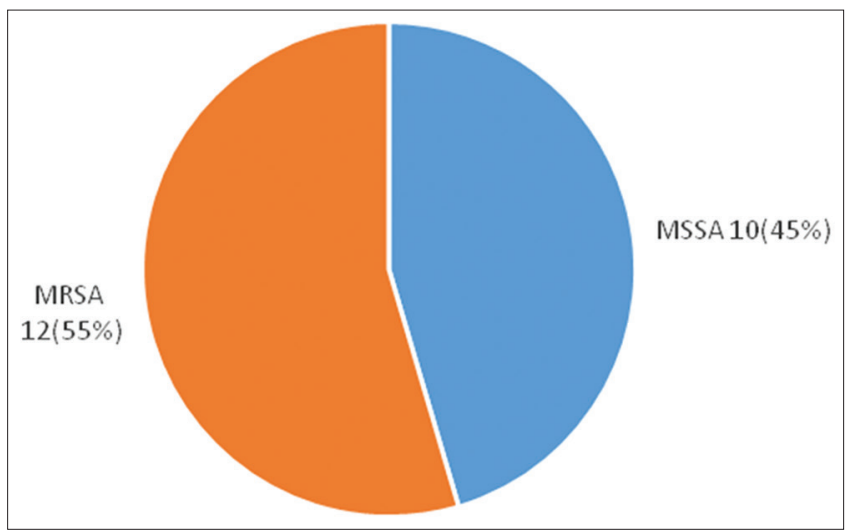

Fig. 2: Percentage of methicillin-resistant Staphylococcus aureus carriers among the $S$, aureus isolates

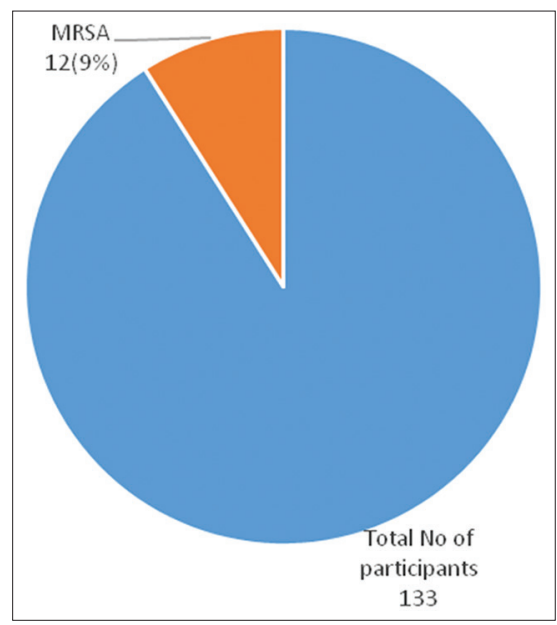

Fig. 3: Percentage of methicillin-resistant Staphylococcus aureus carriers among the total participants 


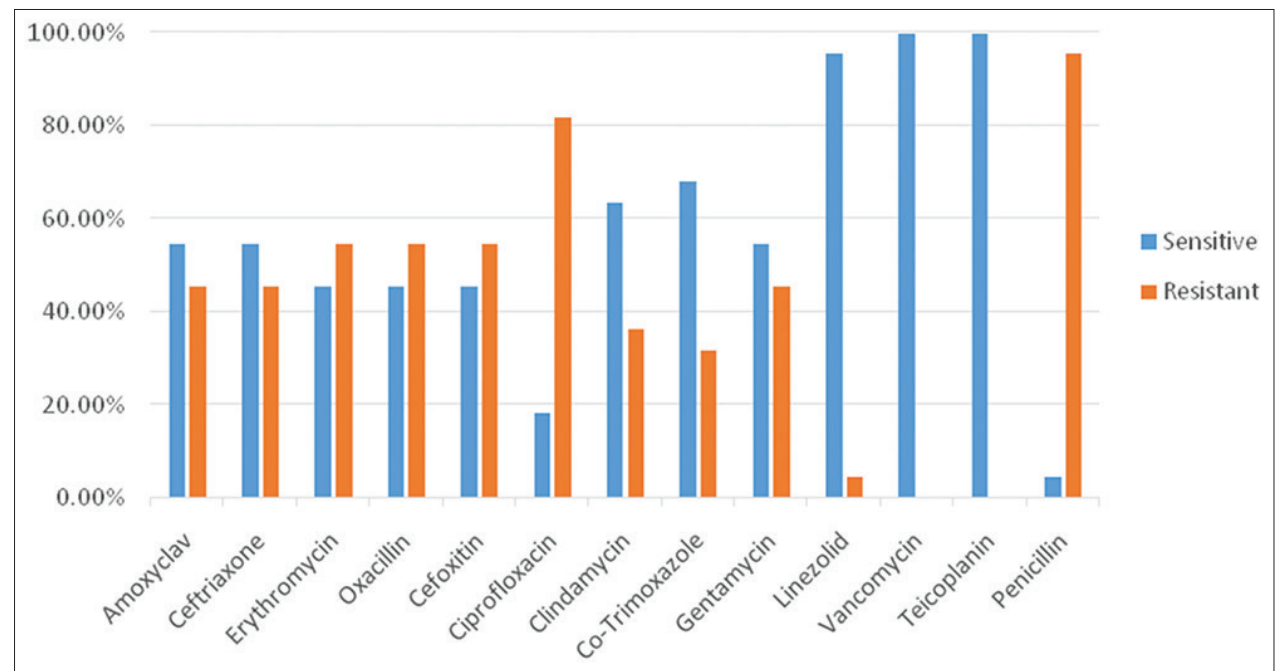

Fig. 4: Antibiotic susceptibility pattern of Staphylococcus aureus isolates

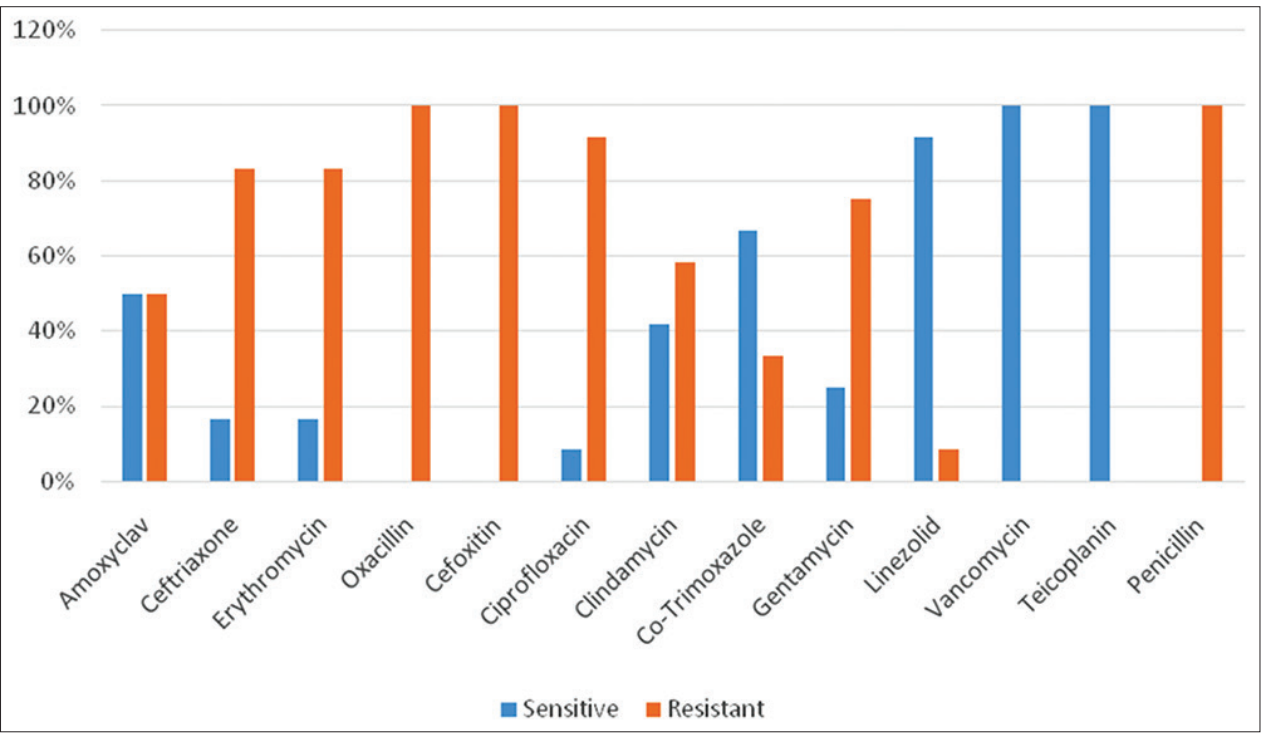

Fig. 5: Antibiotic susceptibility pattern of methicillin-resistant Staphylococcus aureus isolates

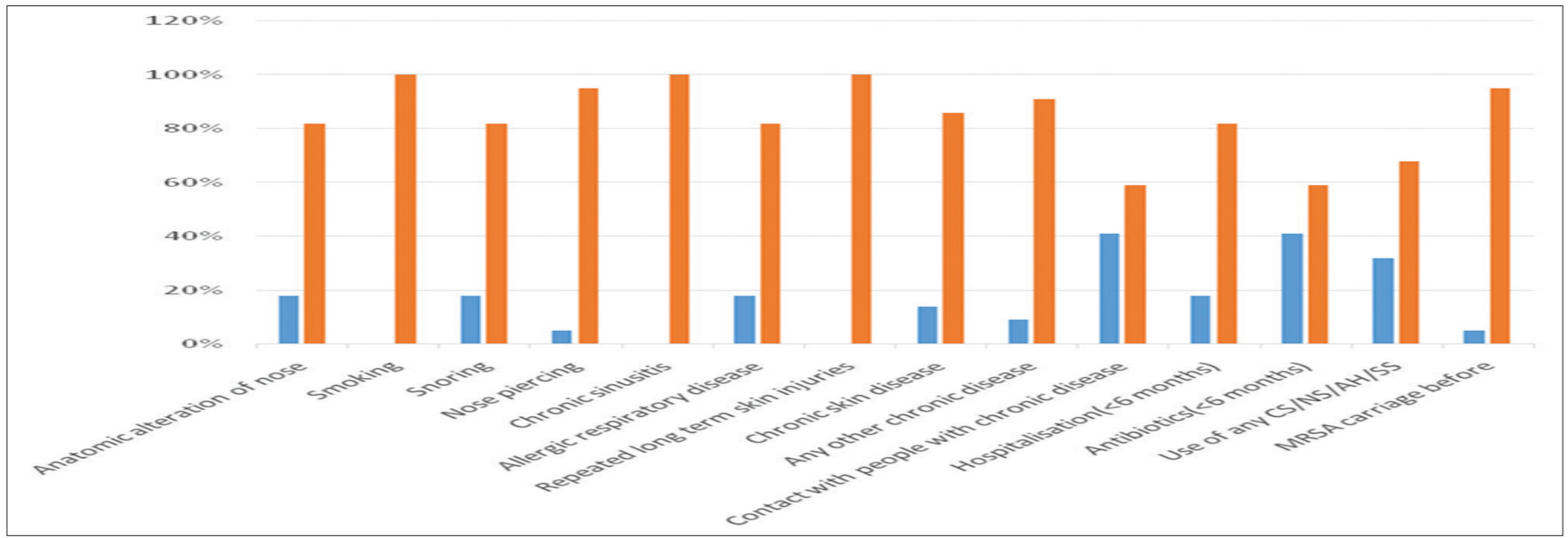

Fig. 6: Relationship between the various parameters and Staphylococcus aureus carriage

of strain, and they are called persistent carriers. A large proportion of the population $(60 \%)$ harbors $S$. aureus intermittently, and the strains change with varying frequencies. Such persons are called intermittent carriers. Finally, minorities of the people (20\%) almost never carry $S$. aureus and they are called noncarriers. The reasons for these dissimilarities in the colonization format are unknown [20]. It is 


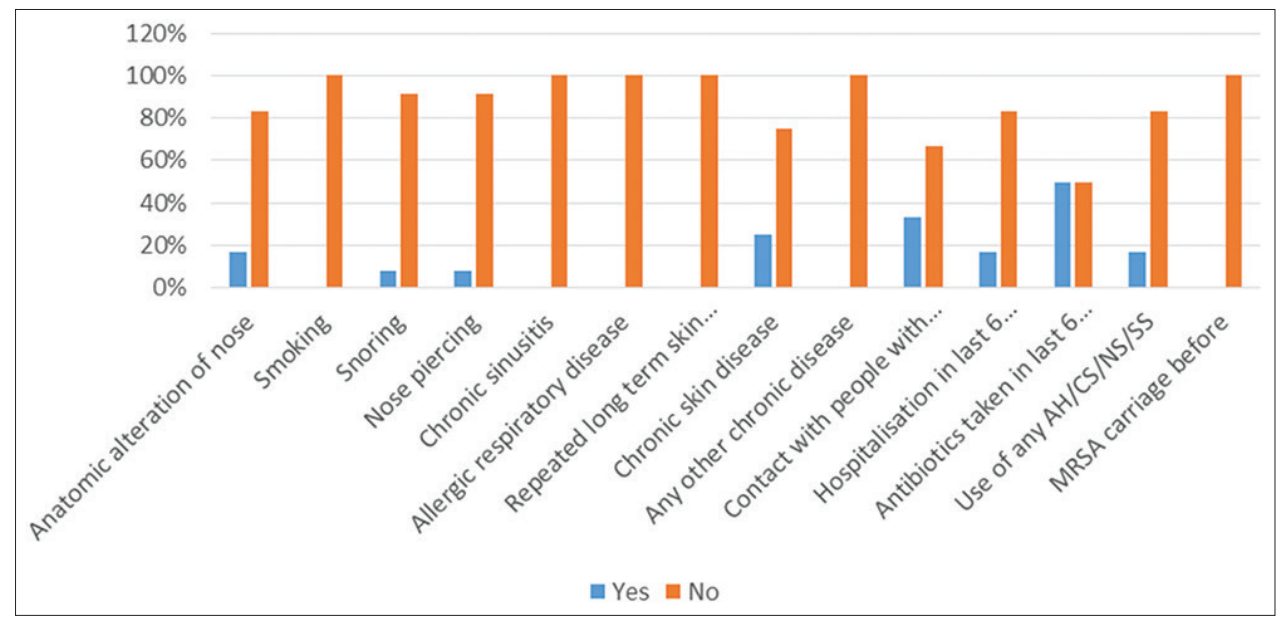

Fig. 7: Relationship between the various parameters and methicillin-resistant Staphylococcus aureus carriage

exciting and necessary to detect the $S$. aureus and MRSA nasal carriage rates as a part of HCWs like clinical postgraduates of medical colleges. At present, there were 241clinical postgraduates, of which 133 had voluntarily participated in the present study representing $55.18 \%$. It was presumed that these individuals might acquire the carriage status of S. aureus/MRSA during their continuous interaction with the patients and thereby may act as a potential source of infection to other patients, resulting in NIs. The prevalence of S. aureus and MRSA varies between institutions and geographic areas. The differences in the study design such as the sample size and the method employed for S. aureus and MRSA detection may account for the disparity in the carriage rate.

According to the result of the present study, the nasal carriage of S. aureus among clinical postgraduates in a medical college of Coastal Karnataka, India, was $16.54 \%$ (22 of 133). Of 22 isolates of $S$. aureus, 12 were MRSA (54.54\%). The S. aureus and MRSA nasal carriage rates of clinical postgraduates recorded in this study were significantly high with the other student population, and this could be due to their continuous exposure with patients in clinical settings [21-24]. A similar kind of research was conducted in our institution past year by involving internees. It was observed that $S$. aureus carriage rate among the said internee's population was $47.3 \%$, the overall carriage rate of MRSA was $1.4 \%$, and among the $S$. aureus carriers, $0.6 \%$ was MRSA [25]. Compared to past year's study, the $S$. aureus carriage rate among the clinical postgraduates was remarkably low (47.3\% and $16.54 \%)$, and MRSA was significantly high $(0.67 \%$ and $9 \%)$, respectively. In all the 12 cases repeated isolation of the MRSA with the same antibiogram pattern concluded that the carriage status was not transient. The study also found that no significant differences in the carriage status of MRSA among the students of various surgical or nonsurgical specialties. Surprisingly, the numbers of MRSA carriers were more in $1^{\text {st }}$-year students when compared with $2^{\text {nd }}$ and final year students. It was observed that among the various risk factors, contact with the chronic patients and consumption of antibiotics in past 6 months found to be statistically significant in relation with nasal carriage status of $S$. aureus/ MRSA $(p<0.005)$. The MRSA was $100 \%$ sensitive to vancomycin and teicoplanin. Aiming at $0 \%$ carriage rate of MRSA among the clinical postgraduates of our institution the identified MRSA carriers may be treated with mupirocin for decolonization and further screened for carriage status to check the efficacy of treatment. However, periodic screening for MRSA should be an on-going practice in the medical student population. This practice can be seen as a task because of this particular group with the highest frequency of contact with the patients and the most likely to transmit this superbug. A further advantage of treating the carriers and institution of effective hospital control policies is in the prevention of its transmission to their family members and others in the community. The most critical factor for preventing NIs is compliance of the health professionals with the sanitary and the antibacterial guidelines, for which the health professionals should be informed about the potential consequences of the NIs, both inside and outside the hospital, and their cooperation should be sought to diminish the carriage of $S$. aureus. Simple preventive measures like hand washing before and after the patient examination, the use of sterile aprons and masks in the post-operative ward, awareness during the investigation of immunocompromised patients, and avoiding touching one's nose during work, can reduce the disease transmission rate considerably. All the HCWs should be periodically educated and trained in the maintenance of hygiene and infection control and the effects of the use or rather, the misuse of antibiotics.

\section{CONCLUSION}

Of 241clinical postgraduates, 133 did participate in the present study. Swabs were collected from the consenting participants after giving a set of questionnaires. The organisms isolated were identified by standard bacteriological methods, and all the isolates of $S$. aureus were further subjected to antibiotics sensitivity testing including methicillin. The numbers of $S$. aureus isolated out of 133 participants were 22(16.5\%). Of 22 isolates of S. aureus, 12 were MRSA (54.5\%). The MRSA carriage rate of clinical postgraduates of Kasturba Medical College, Mangalore, was $9 \%$. Each of the MRSA isolates was sensitive to vancomycin and teicoplanin. Contact with the patients with chronic infections and consumption of antibiotic in past 6 months were found to be statistically significant as the risk factor associated with the acquisition of S. aureus/ MRSA carriage status among the participants. There was no report of vancomycin-resistant $S$. aureus from the present study.

\section{ACKNOWLEDGMENT}

Authors would like to thank the Manipal Academy of Higher Education for having provided facilities for carrying out this research work and the technical staff of the Department of Microbiology KMC., Mangalore. ICMR STS 2014 project had supported this study.

\section{CONFLICTS OF INTEREST}

Nil.

\section{REFERENCES}

1. Foster TJ. The Staphylococcus aureus "superbug." J Clin Invest 2004:114:1693-6.

2. Peacock SJ, de Silva I, Lowy FD. What determines nasal carriage of Staphylococcus aureus? Trends Microbiol 2001;9:605-10.

3. Deresinski S. Methicillin-resistant Staphylococcus aureus: An evolutionary, epidemiologic, and therapeutic odyssey. Clin Infect Dis 2005;40:562-73.

4. Chambers HF. The changing epidemiology of Staphylococcus aureus? Emerg Infect Dis 2001;7:178-82.

5. Salgado CD, Farr BM, Calfee DP. Community-acquired methicillinresistant Staphylococcus aureus: A meta-analysis of prevalence and risk 
factors. Clin Infect Dis 2003;36:131-9.

6. Bischoff WE, Wallis ML, Tucker KB, Reboussin BA, Sherertz RJ. Staphylococcus aureus nasal carriage in a student community: Prevalence, clonal relationships, and risk factors. Infect Control Hosp Epidemiol 2004;25:485-91

7. Albrich WC, Harbarth S. Healthcare workers: Source, vector or victim of MRSA. Lancet Infect Dis 2008;8:289-301.

8. Eveillard M, Martin Y, Hidri N, Boussougant Y, Joly-Guillou ML. Carriage of methicillin-resistant Staphylococcus aureus among hospital employees: Prevalence, duration, and transmission to households. Infect Control Hosp Epidemiol 2004;25:114-20.

9. Prates KA, Torres AM, Garcia LB, Ogatta SF, Cardoso CL, Tognim MC Nasal carriage of methicillin-resistant Staphylococcus aureus in university students. Braz J Infect Dis 2010;14:316-8.

10. Chen CS, Chen CY, Huang YC. Nasal carriage rate and molecular epidemiology of methicillin-resistant Staphylococcus aureus among medical students at a Taiwanese university. Int $\mathbf{J}$ Infect Dis 2012;16:e799-803.

11. BettinA, Causil C, Reyes N. Molecular identification and antimicrobial susceptibility of Staphylococcus aureus nasal isolates from medical students in Cartagena, Colombia. Braz J Infect Dis 2012;16:329-34.

12. Rohde RE, Denham R, Brannon A. Methicillin resistant Staphylococcus aureus: Carriage rates and characterization of students in a Texas university. Clin Lab Sci 2009;22:176-84.

13. Syafinaz AM, Nur Ain NZ, Nadzirahi SN, Fatimah JS, Shahram A, Nasir MD, et al. Staphylococcus aureus nasal carriers among medical students in a medical school. Med J Malaysia 2012;67:636-8.

14. Slifka KJ, Nettleman MD, Dybas L, Stein GE. Is acquisition of methicillin-resistant Staphylococcus aureus an occupational hazard for medical students? Clin Infect Dis 2009;49:482-3.

15. Cheesbrough M. District Laboratory Practice in Tropical Countries. Part 2. Cambridge: Cambridge University Press; 2008. p. 62-70.

16. Bauer AW, Kirby WM, Sherris JC, Turck M. Antibiotic susceptibility testing by a standardized single disk method. Am J Clin Pathol 1966;45:493-6.
17. CLSI. Surveillance for MRSA: Principles, Practices, and Challenges; A Report. CLSI Document X07-R. Wayne, PA: Clinical and Laboratory Standards Institute; 2010.

18. Clinical and Laboratory Standards Institute/NCCLS Performance Standards for Antimicrobial Disc Diffusion Tests; Approved Standards. $9^{\text {th }}$ ed. CLSI Document M2-M9. Wayne Pa: Clinical and Laboratory Standards Institute; 2006.

19. Peacock SJ, Justice A, Griffiths D, De Silva GDI, Kantzanuo MN, Crook D, et al. Determinants of acquisition and carriage of Staphylococcus aureus in Infancy. J Clin Microbiol 2003;41:5718-25.

20. Kluytmans J, van Belkum A, Verbrugh H. Nasal carriage of Staphylococcus aureus: Epidemiology, underlying mechanisms, and associated risks. Clin Microbiol Rev 1997;10:505-20.

21. Santhosh DV, Shobha KL, Bairy I, Rao G, Anand KM, D’Souza J. Nasal screening and survey of pre-clinical medical students from Malaysia for nasal carriage of coagulase positive MRSA and rate of nasal colonization with Staphylococcus species. J Clin Diagn Res 2007; $1: 494-9$

22. Treesirichod A, Hantagool S, Prommalikit O. Nasal carriage and antimicrobial susceptibility of Staphylococcus aureus among medical students at the HRH Princess Maha Chakri Sirindhorn Medical Center, Thailand: A cross-sectional study. J Infect Public Health 2013;6:196-201

23. Ma XX, Sun DD, Wang S, Wang ML, Li M, Shang H, et al. Nasal carriage of methicillin-resistant Staphylococcus aureus among preclinical medical students: Epidemiologic and molecular characteristics of methicillin-resistant Staphylococcus aureus clones. Diagn Microbiol Infect Dis 2011;70:22-30.

24. Zakai SA. Prevalence of methicillin-resistant Staphylococcus aureus nasal colonization among medical students in Jeddah, Saudi Arabia. Saudi Med J 2015;36:807-12.

25. Shreyas K, Radhakrishna M, Hegde A, Rao P. Is acquisition of methicillin-resistant Staphylococcus aureus - An occupational hazard for medical students in India? Asian J Pharm Clin Res 2017;10:141-4. 